\title{
ALMOST-BIEBERBACH GROUPS WITH (IN)FINITE OUTER AUTOMORPHISM GROUP
}

\author{
by WIM MALFAIT* and ANDRZEJ SZCZEPAŃSKI†
}

(Received 23 July, 1996)

\begin{abstract}
If we investigate symmetry of an infra-nilmanifold $M$, the outer automorphism group of its fundamental group (an almost-Bieberbach group) is known to be a crucial object. In this paper, we characterise algebraically almost-Bieberbach groups $E$ with finite outer automorphism group Out $(E)$. Inspired by the description of Anosov diffeomorphisms on $M$, we also present an interesting class of infinite order outer automorphisms. Another possible type of infinite order outer automorphisms arises when comparing $\operatorname{Out}(E)$ with the outer automorphism group of the underlying crystallographic group of $E$.
\end{abstract}

1. Preliminaries. Let us start with some notational remarks used throughout this paper.

1.1. Notational remarks. If $G$ is a group and $g$ is an element of $G$, we write $\mu(g)$ for the conjugation in $G$ determined by $g$; i.e. $\mu(g)(x)=g x g^{-1} \cdot \mu(G)$ is known as the inner automorphism group $\operatorname{Inn}(G)$, while $\operatorname{Out}(G)=\operatorname{Aut}(G) / \operatorname{Inn}(G)$ is called the outer automorphism group of $G$. We write $p: \operatorname{Aut}(G) \rightarrow \operatorname{Out}(G)$ for the natural projection.

$Z(G)$ denotes the center of $G$ and, for a subset $X$ in $G, C_{G} X$ is the centraliser and $N_{C} ; X$ the normaliser of $X$ in $G$. The isolator (or root set) of a subgroup $H$ of $G$ is defined by $\sqrt[i]{H}=\left\{g \in G \| g^{k} \in H\right.$ for some $\left.k \geq 1\right\}$.

If $x, y$ are elements of $G$, we make the following conventions: $[x, y]=x^{-1} y^{-1} x y$ and $x^{y}=y^{-1} x y$. The lower central series of $G$ is defined inductively by $\gamma_{1}(G)=G$ and $\gamma_{n+1}(G)=\left[\gamma_{n}(G), g\right]\left(n \in \mathbb{N}_{0}\right)$. $G$ is said to be $c$-step nilpotent (or nilpotent of class $c$ ) if and only if $\gamma_{c}(G) \neq\{1\}$ and $\gamma_{c+1}(G)=\{1\}$. It then follows that $\gamma_{c}(G) \subseteq Z(G)$.

Each extension $1 \rightarrow G \rightarrow E \rightarrow F \rightarrow 1$ induces, by choosing a normalised section $s: F \rightarrow E$ and by conjugation in $E$, an abstract kernel $\psi: F \rightarrow \operatorname{Out}(G)$. We say that $1 \rightarrow G \rightarrow E \rightarrow F \rightarrow 1$ realises, or is compatible with, $\psi$ (see e.g. [13]).

1.2. (Virtually) unipotent automorphisms of a torsion-free, finitely generated, nilpotent group. Let $N$ be a torsion-free, finitely generated, nilpotent group. Write $L$ for the Mal'cev completion of $N$. Then each automorphism $v$ of $N$ extends uniquely to an automorphism $\tilde{v}$ of $L([\mathbf{1 4}])$. This property is known as the Unique Automorphism Extension Property. Let $I$ indicate the Lie algebra of $L$. I has the same dimension (say $n$ ) and nilpotency class (say $c$ ) as $L$. Moreover, the exponential map exp: $l \rightarrow L$ is bijective

* Postdoctoral Fellow of the Belgian National Fund for Scientific Research (N.F.W.O.)

† This research was partially supported by Polish grant nr.0627/P3/93/04

Glasgow Math. J. 40 (1998) 47-62. 
and we denote its inverse by $\log$. Then, for any automorphism $\tilde{v}: L \rightarrow L$, there exists a unique automorphism $d \tilde{v}: l \rightarrow l$ (the differential of $\tilde{v}$ ) of Lie algebras, making the following diagram commutative

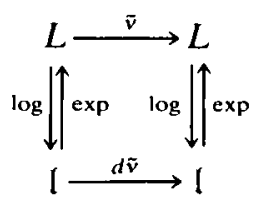

If we now fix a basis of $\mathfrak{l}$ (as $n$-dimensional vector space), each automorphism $v$ of $N$ corresponds to a matrix $A_{v} \in \mathrm{Gl}(n, \mathbb{R})$ (representing $d \tilde{v}$ on l).

An automorphism $v$ of $N$ (or its unique lift $\tilde{v} \in \operatorname{Aut}(L)$ ) is called unipotent if and only if $A_{v}$ is unipotent, i.e. all its eigenvalues are one. In the sequel, we will refer to the eigenvalues of $A_{v}$ as the eigenvalues of $v$ (of $\tilde{v}$ ). In fact, as proved in [3], the set of eigenvalues of $v$ is exactly the collection of the eigenvalues of the induced automorphisms (by considering restrictions and passing through quotients) of the finite dimensional vector spaces $\gamma_{i}(L) / \gamma_{i+1}(L) \cong \mathbb{R}^{k_{i}}$ (where $1 \leq i \leq c$ and $\sum_{i=1}^{c} k_{i}=n$ ) or equivalently; the collection of the eigenvalues of the induced automorphisms of the quotients $\sqrt[N]{\gamma_{i}(N)} / \sqrt[N]{\gamma_{i+1}(N)} \cong \mathbb{Z}^{k_{i}}(1 \leq i \leq c)$.

An interesting result is the following:

Lemma 1.1. [17, Lemma 2]. If $v$ is an automorphism of a torsion-free, finitely generated, nilpotent group $N$ which induces a unipotent automorphism on $N / \sqrt[N]{\gamma_{2}(N)}$, then $v$ is unipotent.

This immediately implies that inner automorphisms of $N$ are unipotent.

An automorphism $v$ of $N$ (or its unique lift $\tilde{v} \in \operatorname{Aut}(L)$ ) is called virtually unipotent if and only if there is a power of $A_{v}$ which is unipotent. Obviously, we have

LEMMA 1.2. If $v$ is an automorphism of a torsion-free, finitely generated, nilpotent group $N$ which induces a virtually unipotent automorphism on $N / \sqrt[N]{\gamma_{2}(N)}$, then $v$ is virtually unipotent.

Note that all eigenvalues of a virtually unipotent automorphism $v$ of $N$ are of absolute value one (since all eigenvalues of a unipotent matrix are one). In this context (not in general however), also the converse holds since all coefficients of the characteristic polynomial of $A_{v}$ are integers and hence all eigenvalues are roots of unity (e.g. [1, proof of Prop. 2.2], [19, Lemma 11.6]). Consequently, an automorphism of $N$ is virtually unipotent if and only if all its eigenvalues are of absolute value one.

2. Introduction. First, we review the fundamentals of an algebraic approach, which was the subject of the Ph.D. thesis of W. Malfait ([15]), to study symmetry of infra-nilmanifolds and explain, from this point of view, our interest in almost- Bieberbach groups with finite outer automorphism group. 
2.1. Symmetry of infra-nilmanifolds: an algebraic approach. Let $L$ be a connected and simply connected, nilpotent Lie group. We write $\operatorname{Aff}(L)=L \rtimes \operatorname{Aut}(L)$ for the group of affine diffeomorphisms of $L$ with group law $(l, \alpha) \cdot\left(l^{\prime}, \beta\right)=\left(l \cdot \alpha\left(l^{\prime}\right), \alpha \circ \beta\right)$. Aff $(L)$ acts in a natural way on $L$; for $x, y \in L$ and $\alpha \in \operatorname{Aut}(L),(x, \alpha) \cdot y=x \alpha(y)$. Let $C$ be a maximal compact subgroup of $\operatorname{Aut}(L)$. An almost-crystallographic group is a discrete and uniform subgroup $E$ of the semi-direct product $L \rtimes C \subset L \rtimes \operatorname{Aut}(L)=\operatorname{Aff}(L)$. The translation subgroup $N=E \cap L$ is a lattice of $L$ and is the unique normal subgroup of $E$ which is maximal nilpotent. The quotient $F=E / N$, which is called the holonomy group, is a finite group acting faithfully on $L$. If $E$ is torison-free, then $E$ is an almost-Bieberbach group and the corresponding compact orbit space $M=E \backslash L$ is an infra-nilmanifold. Therefore, almost-Bieberbach groups are exactly the fundamental groups of infranilmanifolds. Clearly, this set-up is a straightforward generalisation of the classical theory of Bieberbach groups and flat Riemannian manifolds (i.e. the situation $L=\mathbb{R}^{\prime \prime}$ ).

Write $\operatorname{Aff}(M)$ for the group of affine diffeomorphisms of an infra-nilmanifold $M$ onto itself or equivalently, $\operatorname{Aff}(M)$ is the group of diffeomorphisms of $M$ whose liftings to its universal cover $L$ belong to $\operatorname{Aff}(L)$. In fact, $\operatorname{Aff}(M)$ can also be seen as the group of diffeomorphisms of $M$ arising by conjugation in $N_{\text {Affi }(L)} E$ (via an epimorphism $q: N_{\text {Aff }(l)} E \rightarrow \operatorname{Aff}(M)$ with kernel $\left.E\right)$. Write $\Phi: \operatorname{Aff}(M) \rightarrow \operatorname{Out}(E)$ for the natural homomorphism defined as follows: conjugating $E(\subset \operatorname{Aff}(L))$ with a homeomorphism $f$ in $N_{\text {Afl }(I .)} E$ induces an $E$-automorphism which represents $\Phi(q(f)) \in \operatorname{Out}(E)$. Due to the work of K. B. Lee and F. Raymond ([12]), the classical second Bieberbach theorem was generalised towards almost-crystallographic groups, stating that each automorphism of $E$ can be realised as affine conjugation in $\operatorname{Aff}(L)$. Hence, $\Phi$ is surjective. It is also known that $C_{\mathrm{AIr}\left(L_{)}\right)}(E)$ is isomorphic to $L^{F}$, the set of points fixed by the canonical action of the holonomy $F$ on $L$ ([10]). Consequently, we have the following commutative diagram built with exact rows and columns:

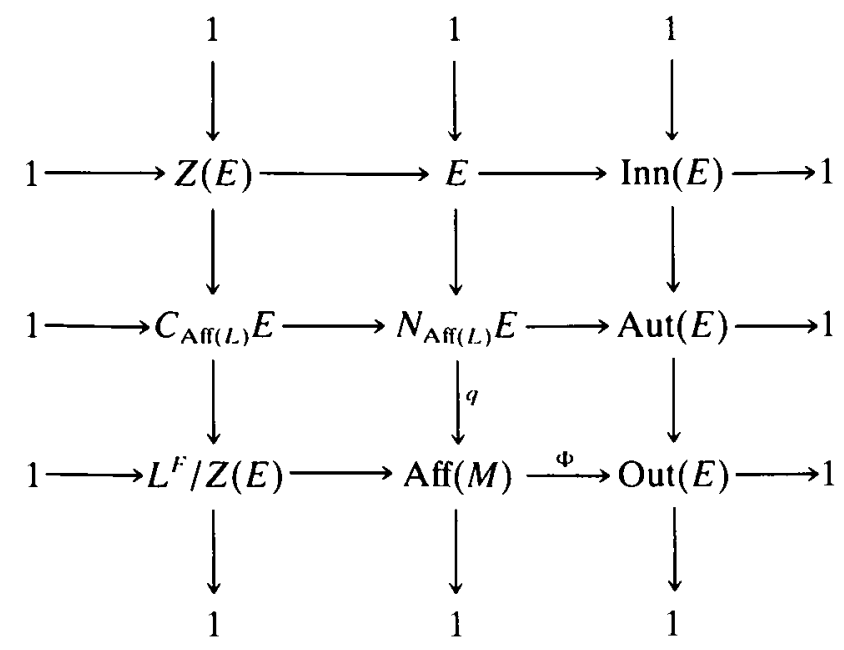

We think of symmetry of an infra-nilmanifold in terms of finite groups acting effectively on it as a group of affine diffeomorphisms. A finite subgroup $F$ of the outer automorphism group of an almost-Bieberbach group $E$ acts effectively as a group of affine diffeomorphisms on the corresponding infra-nilmanifold $M$ if and only if this 
faithful, finite abstract kernel $F \rightarrow \operatorname{Out}(E)$ can be realised by an extension $([\mathbf{1 0}])$. This is of course of major importance for this topologically inspired topic since it allows to convert the study of this kind of symmetry aspects of the infra-nilmanifold $M$ into an algebraic problem of realising faithful, finite abstract kernels by a group extension.

If $L^{F}$ is trivial (we refer the reader to [9] for an equivalent algebraic condition), then every finite group acting effectively on the infra-nilmanifold $M$ as a group of affine diffeomorphisms, is projected isomorphically in $\operatorname{Out}(E)$ by $\Phi$. Hence, the finite subgroups of $\operatorname{Out}(E)$ will control the symmetry of $M$. In general however, if $L^{F}$ is not trivial, our approach (via the finite subgroups of $\operatorname{Out}(E)$ ) is only a partial step towards a complete study of all finite, effective group actions on the infra-nilmanifold $M$. In fact, if there is a non-trivial center, say of rank $k$, in $E$, there is a $k$-dimensional torus $Z(L)^{F} / Z(E)$ acting effectively on $M([10])$.

Even if $L^{F}=\{1\}$, a complete survey of all symmetry aspects of an infra-nilmanifold $M$ is only possible if we can classify (up to conjugation) all finite subgroups of the outer automorphism group of the almost-Bieberbach group $E$. In case Out $(E)$ is infinite, in general such a classification is extremely hard to obtain. Therefore, we look for almost-Bieberbach groups with finite outer automorphism group and try to characterise them (or outer automorphisms of infinite order) algebraically.

2.2. Main results. Our first objective is to generalise a well known result for Bieberbach groups stating that a $n$-dimensional Bieberbach group has only finitely many outer automorphisms if and only if the centraliser of its holonomy in $\operatorname{Gl}(n, \mathbb{Z})$ is finite ([20]). In fact, we will show that an almost-Bieberbach group has only finitely many outer automorphisms if and only if the centraliser of its holonomy group in the outer automorphism group of its translation subgroup is finite (Theorem 3.2).

When working with nilpotency, reduction to the abelian situation is frequently used. In this perspective we will prove that, when the underlying crystallographic group $\operatorname{Cr}(E)$ of an almost-Bieberbach group $E$ has only finitely many outer automorphisms, then Out $(E)$ can only be infinite if and only if there exists a well determined kind of unipotent automorphisms of its translation subgroup $N$ having infinite order in $\operatorname{Out}(N)$ (Theorem 4.4). Conversely, we present a sufficient condition (also in Theorem 4.4) to have that $\operatorname{Out}(E)$ is finite implies that $\operatorname{Out}(\operatorname{Cr}(E))$ is finite. Here the authors want to remark that they believe that this condition is much too strong. However they are not able to find a weaker one. It is even an open problem to find an almost-crystallographic group $E$ with finite outer automorphism group but such that $\operatorname{Out}(\operatorname{Cr}(E))$ is infinite.

We also indicate another possible class of infinite order outer automorphisms of an almost-Bieberbach group $E$. Namely, an automorphism of $E$, restricting to an automorphism of its translation subgroup which is not virtually unipotent, induces an infinite order element in $\operatorname{Out}(E)$ (and also in $\operatorname{Out}(\operatorname{Cr}(E))$ ) (Theorem 5.4). As an interesting consequence, this will imply that the outer automorphism group of the fundamental group of an infra-nilmanifold supporting an Anosov diffeomorphism is always infinite (Corollary 5.7).

Finally, the authors want to remark that it is still an open problem (closely related to the one stated above) to discover and describe other possible classes of infinite order outer automorphisms of an almost-Bieberbach group or perhaps, to prove that the two types discussed in this paper cover all possibilities. 
3. Extensions realising a faithful finite abstract kernel and having only finitely many outer automorphisms. In [8], for group extensions $1 \rightarrow N \rightarrow E \rightarrow F \rightarrow 1$ realising a faithful abstract kernel $F \rightarrow \operatorname{Out}(N)$, commutative diagrams were developed to describe $\operatorname{Aut}(E, N)$ (the group of all $E$-automorphisms mapping $N$ onto itself) and $\operatorname{Out}(E, N)=$ $\operatorname{Aut}(E, N) / \operatorname{lnn}(E)$.

The initiating cases to us were the almost-crystallographic groups. As abstract group, a group $E$ is almost-crystallographic if and only if $E$ contains a finitely generated, torsion-free, nilpotent normal subgroup $N$ of finite index, which is maximal nilpotent in $E$ ([11]). The corresponding extension $1 \rightarrow N \rightarrow E \rightarrow F \rightarrow 1$ is called essential. A basic fact concerning these extensions is that an essential extension induces a faithful abstract kernel ([5]). Consequently, the theory developed in [8] can be applied and moreover, since $N$ is characteristic in $E$, we are automatically concerned with the complete (outer) automorphism group of $E$.

Let us recall the theoretic set-up to construct the fundamental automorphism diagrams mentioned above. For more details, we refer the reader to [8]. Let $\psi: F \rightarrow$ $\operatorname{Out}(N)$ be a faithful abstract kernel. Assume $1 \rightarrow N \rightarrow E \rightarrow F \rightarrow 1$ is a group extension compatible with $\psi$ and determining a cohomology class $a \in H_{\psi}^{2}(F, N)\left(H_{\psi}^{2}(F, N)\right.$ denotes the set of 2-cohomology classes compatible with $\psi$ ).

We define a subgroup $\mathcal{M}_{\psi}$ of $\operatorname{Aut}(N)$ by $\mathcal{M}_{\psi}=p^{-1}\left(N_{\text {Out }(N)} \psi(F)\right)$, the inverse image in $\operatorname{Aut}(N)$ of the normaliser of $\psi(F)$ in $\operatorname{Out}(N)$. We write ${ }^{*}: M_{\psi} \rightarrow \operatorname{Aut}(F)$ for the corresponding group homomorphism, defined by $v^{*}=\psi^{-1} \circ \mu(p(v))^{\circ} \psi$, for each $v \in M_{\psi}$. There is a group action of $M_{\psi}$ on $H_{\psi}^{2}(F, N)$. Write $M_{\psi, a}$ for the stabiliser of $a$ under the action of $\mathcal{M}_{\psi}$ on $H_{\psi}^{2}(F, N)$. Remark that the index of $\mathcal{M}_{\psi, a}$ in $\mathcal{M}_{\psi}$ is of course smaller or equal than the order of $H_{\psi}^{2}(F, N)$ (which equals the order of $H^{2}(F, Z(N))$ if $\psi$ can be realised $([\mathbf{1 3}]))$.

An automorphism $\sigma$ in $\operatorname{Aut}(E, N)$ restricts to an automorphism of $N$ via a homomorphism $A: \operatorname{Aut}(E, N) \rightarrow \operatorname{Aut}(N)$. This induces short exact sequences

$$
1 \rightarrow Z_{\psi}^{\prime}(F, Z(N)) \rightarrow \operatorname{Aut}(E, N) \stackrel{A}{\rightarrow} M_{\psi, a} \rightarrow 1
$$

and

$$
1 \rightarrow H_{\psi}^{\prime}(F, Z(N)) \rightarrow \operatorname{Out}(E, N) \rightarrow Q \rightarrow 1 .
$$

The quotient $Q$ equals

$$
Q=\overline{\mathcal{M}_{\psi, l}} / F \quad \text { where } \quad \overline{M_{\psi, a t}}=\mathcal{M}_{\psi, a} / \operatorname{Inn}(N)
$$

and fits into an extension

$$
1 \rightarrow Q_{2}=\left(\overline{\operatorname{Ker}(*) \cap M_{\psi, a}}\right) / Z(F) \rightarrow Q \rightarrow Q_{1}=M_{\psi . a}^{*} / \operatorname{Inn}(F) \rightarrow 1 .
$$

Here $\mathscr{M}_{\phi, a}^{*}$ denotes the image of $\mathscr{M}_{\psi, a}$ under $*$,

$$
\left(\overline{\operatorname{Ker}(*) \cap M_{\psi, a}}\right)=\left(\operatorname{Ker}\left(^{*}\right) \cap M_{\psi, a}\right) / \operatorname{Inn}(N)
$$

and this is contained in

$$
\left(\operatorname{Ker}(*) \cap \cdot M_{\psi}\right) / \operatorname{Inn}(N)=C_{\text {Out }(N)} \psi(F) .
$$

This allows to prove, for an extension compatible with a faithful and finite abstract 
kernel, the following algebraic criterion (formulated only in terms of the abstract kernel!) to have only finitely many outer automorphisms.

Proposition 3.1. Let $\psi: F \rightarrow \operatorname{Out}(N)$ be a faithful and finite abstract kernel. Assume $Z(N)$ is finitely generated and $1 \rightarrow N \rightarrow E \rightarrow F \rightarrow 1$ is an extension realising $\psi$. Then $\operatorname{Out}(E, N)$ is finite if and only if $N_{\mathrm{Out}(N)} \psi(F)$ is finite if and only if $C_{\mathrm{Out}(N)} \psi(F)$ is finite.

Proof. Since $F$ is finite (which will be used several times in this proof without mentioning it explicitly) and $Z(N)$ is finitely generated, extension (2) has a finite kernel and hence, $\operatorname{Out}(E, N)$ is finite if and only if $Q$ is finite.

Therefore, if $\operatorname{Out}(E, N)$ is finite, then because of (3), $\overline{M_{\psi, a}}=\mathcal{M}_{\psi, a} / \operatorname{Inn}(N)$ is also finite. But $\mathcal{M}_{\psi \cdot a}$ is of finite index in $\mathcal{M}_{\psi}$ and hence, $\mathcal{M}_{\psi} / \operatorname{Inn}(N)=N_{\text {Out }(N)} \psi(F)$ is finite.

Assume $C_{\mathrm{Out}(N)} \psi(F)$ is finite. Becuase of (5), $Q_{2}$ is finite. Since $Q_{1}$ is also finite, it follows that $Q$, or equivalently $\operatorname{Out}(E, N)$, is finite (use (4)).

In particular, for almost-Bieberbach groups this means we have

THEOREM 3.2. The fundamental group of an infra-nilmanifold (i.e. an almostBieberbach group) has only finitely many outer automorphisms if and only if the centraliser of its holonomy group in the outer automorphism group of its translation subgroup is finite.

This clearly generalises the well known ([20]) similar result for Bieberbach groups.

4. Reduction to almost-crystallographic groups of lower dimension. Given an extension $1 \rightarrow N \rightarrow E \rightarrow F \rightarrow 1$ realising a faithful finite abstract kernel and a characteristic subgroup $\Gamma$ of $N$, we can factor out $\Gamma$ to obtain $1 \rightarrow N / \Gamma \rightarrow E / \Gamma \rightarrow F \rightarrow 1$. In this section, we investigate the relation between the (in)finiteness of $\operatorname{Out}(E)$ and $\operatorname{Out}(E / \Gamma)$ in case the induced extension of quotients also is compatible with a faithful abstract kernel.

4.1. Factoring out a characteristic subgroup. Each automorphism $v \in \operatorname{Aut}(N)$ induces automorphisms $C(v)$ of $\Gamma$ and $D(v)$ of $N / \Gamma$ such that the diagram below is commutative:

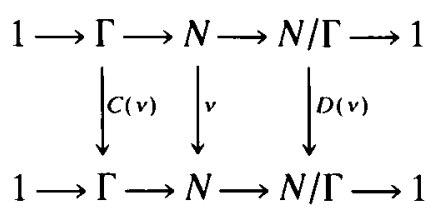

Write $D:$ Aut $(N) \rightarrow \operatorname{Aut}(N / \Gamma)$ for the homomorphism obtained by passing through the quotient and $\bar{D}: \operatorname{Out}(N) \rightarrow \operatorname{Out}(N / \Gamma)$ for the induced homomorphism on outer automorphism level. Let us introduce the following notion:

Definition 4.1. Let $N$ be a group. A subgroup $\Gamma$ of $N$ is called super-characteristic if and only if $\Gamma$ is characteristic in $N$ and the image of $D: \operatorname{Aut}(N) \rightarrow \operatorname{Aut}(N / \Gamma)$ has finite index in $\operatorname{Aut}(N / \Gamma)$.

For example, in case $D$ is surjective, then $\Gamma$ is super-characteristic. In the sequel, we also need the following elementary group-theoretical result: 
LeMmA 4.2. Let $G$ be a group, $X$ a subgroup of $G$ and $N$ a normal subgroup of $G$. Then $C_{G}^{N} X=\{g \in G \| \forall x \in X[g, x] \in N\}$ is a subgroup of $G$ and

1. $C_{G}^{N} X / N=C_{G / N} X / X \cap N$.

2. The index of $C_{G} X$ in $C_{G}^{N} X$ is smaller than or equal to the cardinality of $N^{X}$, the set of all mappings of $X$ into $N$.

Proof. Obviously, since $N$ is normal in $G, C_{G}^{N} X$ is a subgroup of $G$ and, when factoring out $N$, we obtain the centraliser of $X / X \cap N$ in $G / N$.

Now, define a map

$$
\begin{aligned}
\tau: C_{G}^{N} X / C_{G} X & \rightarrow N^{X} \\
g C_{G} X & \mapsto n_{g}: X \rightarrow N: x \mapsto[g, x]
\end{aligned}
$$

Assume $n_{g}=n_{h}$, for some $g, h$ in $G$, or, for all $x$ in $X,[g, x]=[h, x]$. Then

$$
\forall x \in X\left[g h^{-1}, x\right]=[g, x]^{h^{-1}}\left[h^{-1}, x\right]=\left([g, x][h, x]^{-1}\right)^{h^{-1}}=1
$$

or $g C_{G} X=h C_{G} X$. This shows that $\tau$ is injective.

Now, we can formulate the following result.

Proposition 4.3. Let $1 \rightarrow N \rightarrow E \rightarrow F \rightarrow 1$ be an extension realising a faithful, finite abstract kernel $\psi: F \rightarrow \operatorname{Out}(N)$. Assume $\Gamma$ is characteristic in $N$. Consider $D: \operatorname{Aut}(N) \rightarrow$ $\operatorname{Aut}(N / \Gamma)$ and $\bar{D}: \operatorname{Out}(N) \rightarrow \operatorname{Out}(N / \Gamma)$ as introduced above. Assume the abstract kernel $\psi_{1}=\bar{D} \circ \psi: F \rightarrow \operatorname{Out}(N / \Gamma)$, induced by the extension of quotients $1 \rightarrow N / \Gamma \rightarrow E / \Gamma \rightarrow F \rightarrow$ 1 , is faithful. Define a subgroup $T$ of $\mathcal{M}_{\psi}$, the inverse image in $\operatorname{Aut}(N)$ of the normaliser of $\psi(F)$ in $\operatorname{Out}(N)$, as

$$
\begin{aligned}
T & =\left\{v \in M_{\psi} \| D(v) \in \operatorname{Inn}(N / \Gamma) \text { and } v \in \operatorname{Ker}(*)\right\} \\
& =p^{-1}\left(\operatorname{Ker}(\bar{D}) \cap C_{\mathrm{Oul}(N)} \psi(F)\right)
\end{aligned}
$$

the inverse image in $\operatorname{Aut}(N)$ of the intersection of the centraliser of $\psi(F)$ in $\operatorname{Out}(N)$ and the kernel of $\bar{D}$.

1. If $\operatorname{Out}(E / \Gamma, N / \Gamma)$ is finite, then $\operatorname{Out}(E, N)$ is finite if and only if $\operatorname{Inn}(N)$ has finite index in $T$.

2. If $\Gamma$ is super-characteristic in $N$ and $\operatorname{Ker}(\bar{D})$ is finite, then $\operatorname{Out}(E, N)$ is finite implies that $\operatorname{Out}(E / \Gamma, N / \Gamma)$ is finite.

Proof. As $\psi_{1}=\bar{D} \circ \psi$ is injective, $\psi(F) \cap \operatorname{Ker}(\bar{D})$ is trivial and the homomorphism $\bar{D}$ determines the following commutative diagram of short exact sequences (use Lemma 4.2):

$$
\begin{aligned}
& 1 \rightarrow T / \operatorname{Inn}(N) \rightarrow C_{\mathrm{Out}(N)} \psi(F) \stackrel{\bar{D}}{\rightarrow} \bar{D}\left(C_{\mathrm{Out}(N)} \psi(F)\right) \rightarrow 1 \\
& \cap \cap \cap \cap \\
& 1 \rightarrow \operatorname{Ker}(\bar{D}) \rightarrow C_{\mathrm{Out}(N)}^{\operatorname{Kur}(\bar{D})} \psi(F) \stackrel{\bar{D}}{\rightarrow} C_{\mathrm{Im}(\bar{D})} \psi_{\mathrm{r}}(F) \quad \rightarrow 1 \\
& \cap \\
& C_{\text {Out(NI) })} \psi_{\Gamma}(F)
\end{aligned}
$$


Applying Proposition 3.1 several times, we easily deduce the first result. If $\operatorname{Out}(E, N)$ is finite, or $C_{\operatorname{Out}(N)} \psi(F)$ is finite, then $\operatorname{Inn}(N)$ has finite index in $T$. If $\operatorname{Out}(E / \Gamma, N / \Gamma)$ (or equivalently $\left.C_{\mathrm{Out}(N / \mathrm{T})} \psi_{\mathrm{J}}(F)\right)$ and $p(T)$ are finite, then $C_{\mathrm{Out}(N)} \psi(F)(\operatorname{or} \operatorname{Out}(E, N))$ is clearly finite.

The second result is not that straightforward. Assume $\operatorname{Ker}(\bar{D})$ is finite. Then the index of $C_{\operatorname{Out}(N)} \psi(F)$ in $C_{\operatorname{Out}(N)}^{\operatorname{Ker}(\bar{D})} \psi(F)$ is finite since it is smaller or equal than the cardinality of the set of mappings of the finite group $F$ into $\operatorname{Ker}(\bar{D})$ (Lemma 4.2). Hence, if $\operatorname{Out}(E, N)$ (or $\left.C_{\operatorname{Our}(N)} \psi(F)\right)$ is also finite, then $C_{\operatorname{Im}(\bar{D})} \psi_{\Gamma}(F)$ is finite. If $\Gamma$ is super-characteristic in $N$, then $\operatorname{Im}(\bar{D})$ has finite index in $\operatorname{Out}(N / \Gamma)$ and hence, also $C_{\text {Out }(N / \Gamma)} \psi_{\Gamma}(F)($ or $\operatorname{Out}(E / \Gamma, N / \Gamma))$ is finite.

4.2. Reduction to the underlying crystallographic group. Let us return to our main point of interest and translate the results stated above into the language of almostcrystallographic groups. Let $E$ be an almost-crystallographic group of a Lie group $L$ and fitting into an essential extension $1 \rightarrow N \rightarrow E \rightarrow F \rightarrow 1$. The nilpotent kernel $N$ (say of class $c$ ) is the translation subgroup of $E$ and $L$ is its Mal'cev completion. When working with nilpotency, induction from the abelian situation or reduction to a lower nilpotency class is very common. In this perspective, Proposition 4.3 can be useful.

Indeed, some characteristic subgroups $\Gamma$ of $N$ are known such that the resulting extension (of quotients) again is essential (or $E / \Gamma$ is almost-crystallographic and the corresponding abstract kernel $F \rightarrow \operatorname{Out}(N / \Gamma)$ is injective) and with $N / \Gamma$ of lower nilpotency class. In fact, as seen in [9], at least the following choices are available: taking $\Gamma=\sqrt[N]{\gamma_{i+1}(N)}(1 \leq i \leq c)$ will reduce the nilpotency class exactly to $i$-step nilpotency and gives rise to an almost-crystallographic group $E / \sqrt[N]{\gamma_{i+1}(N)}$ of the Lie group $L / \gamma_{i+1}(L)$.

In case $\Gamma=\sqrt[N]{\gamma_{2}(N)}$, the nilpotency class is reduced substantially and brings us to an abelian kernel situation. The quotient $E / \Gamma$ is called the underlying crystallographic group $\operatorname{Cr}(E)$ of $E$. Proposition 4.3 can now be formulated as follows:

THEOREM 4.4. Let $E$ be an almost-crystallographic group with translation subgroup $N$ and holonomy group $F$. Write $\Gamma$ for the isolator $\sqrt[N]{\gamma_{2}(N)}$ of the commutator subgroup of $N$ in $N$ and $D: \operatorname{Aut}(N) \rightarrow \operatorname{Aut}(N / \Gamma)\left(\cong \mathrm{Gl}(n, \mathbb{Z})\right.$ for some $\left.n \in \mathbb{N}_{0}\right)$ for the homomorphism obtained by passing through the quotient. Then

1. If the underlying crystallographic group $\operatorname{Cr}(E)$ of $E$ has only finitely many outer automorphisms, then $\operatorname{Out}(E)$ is finite if and only if $\operatorname{Inn}(N)$ has finite index in $T=\operatorname{Ker}(D) \cap \operatorname{Ker}(*)$.

2. If $\Gamma$ is super-characteristic in $N$ and $\operatorname{Inn}(N)$ has finite index in $\operatorname{Ker}(D)$, then $\operatorname{Out}(E)$ is finite implies that $\operatorname{Cr}(E)$ has only finitely many outer automorphisms (or equivalently; $C_{\mathrm{Gl}(n, \mathbb{Z})} F$ is finite).

REMARK 4.5.

(i) Since $N$-automorphisms in $T$ induce the identity on $N / \sqrt[N]{\gamma_{2}(N)}$, they are unipotent (Lemma 1.1). We remark that in [6] a class of almost-crystallographic groups having infinite order outer automorphisms, restricting (on the translation subgroup) to such unipotent automorphisms as discussed here, is indicated.

(ii) As already mentioned in the introduction, we invite the reader to weaken the sufficient condition to have that $\operatorname{Out}(E)$ is finite implies that $\operatorname{Out}(\operatorname{Cr}(E))$ is finite. A first objective should be the construction of an almost-crystallographic group 
$E$ with only finitely many outer automorphisms and such that there is an outer automorphism of $\operatorname{Cr}(E)$ of infinite order.

4.3. Examples. We illustrate our results using low-dimensional ( $\leq 4)$ almostBieberbach groups. For an isomorphism type classification of these groups, we refer the reader to $[2]$ and $[\mathbf{4}]$.

4.3.1. 3-dimensional almost-crystallographic groups. It is well known that every finitely generated, torsion-free, nilpotent group of rank 3 is isomorphic to a Heisenberg type group, $\Gamma_{k}$, for some $k \in \mathbb{N}_{0}$ :

$$
\Gamma_{k}:\left\langle a, b, c \|[b, a]=c^{k},[c, a]=[c, b]=1\right\rangle .
$$

Consequently, each 3-dimensional almost-crystallographic group $E$ fits into an essential extension $1 \rightarrow \Gamma_{k} \rightarrow E \rightarrow F \rightarrow 1$. The characteristic subgroup $\Gamma=\sqrt[\Gamma_{k}]{\gamma_{2}\left(\Gamma_{k}\right)}$ of $\Gamma_{k}$ is the subgroup generated by $c$. To study $\operatorname{Aut}\left(\Gamma_{k}\right)$, think of

$$
1 \rightarrow \Gamma \cong \mathbb{Z} \rightarrow \Gamma_{k} \rightarrow \Gamma_{k} / \Gamma \cong \mathbb{Z}^{2} \rightarrow 1
$$

Each automorphism $v$ in $\operatorname{Aut}\left(\Gamma_{k}\right)$ restricts to an automorphism of $\mathbb{Z}$, and consequently induces, via $D: \operatorname{Aut}\left(\Gamma_{k}\right) \rightarrow \operatorname{Aut}\left(\mathbb{Z}^{2}\right)$, an automorphism of $\mathbb{Z}^{2}$. As $v$ must respect the relations given above, one verifies that $v$ sends $a \mapsto a^{v_{1}} b^{\nu_{3}} c^{1_{1}}, b \mapsto a^{\nu_{2}} b^{\nu_{4}} c^{I_{2}}$ and $c \mapsto c^{D_{v}}$, where $l_{1}, l_{2} \in \mathbb{Z},\left(\begin{array}{ll}v_{1} & v_{2} \\ v_{3} & v_{4}\end{array}\right) \in \mathrm{Gl}(2, \mathbb{Z})$ and $D_{v}=v_{1} v_{4}-v_{2} v_{3}= \pm 1$. Obviously, $D$ is onto, or $\Gamma$ is certainly super-characteristic in $\Gamma_{k}$, and $\operatorname{Ker}(D)$ contains all automorphisms $v_{\left(l_{1}, l_{2}\right)}$ mapping $a \mapsto a c^{\prime_{1}}$ and $b \mapsto b c^{l_{2}}$, for some $l_{1}, l_{2} \in \mathbb{Z}$. Hence, there is an exact sequence

$$
1 \rightarrow \mathbb{Z}^{2} \rightarrow \operatorname{Aut}\left(\Gamma_{k}\right) \stackrel{D}{\rightarrow} \mathrm{Gl}(2, \mathbb{Z}) \rightarrow 1 .
$$

An inner automorphism $\mu\left(a^{\alpha_{1}} b^{\alpha_{2}} c^{\alpha_{3}}\right): \Gamma_{k} \rightarrow \Gamma_{k}\left(\alpha_{i} \in \mathbb{Z}\right)$ sends $a \mapsto a c^{k \alpha_{2}}$ and $b \mapsto b c^{-k \alpha_{1}}$ and consequently, $\operatorname{Ker}(D) / \operatorname{Inn}\left(\Gamma_{k}\right) \cong \mathbb{Z}_{k} \times \mathbb{Z}_{k}$. We conclude that we have

COROLlary 4.6. Let E be a 3-dimensional almost-crystallographic group fitting into an essential extension $1 \rightarrow \Gamma_{k} \rightarrow E \rightarrow F \rightarrow 1$. Then $\operatorname{Out}(E)$ is finite if and only if $\operatorname{Out}(\operatorname{Cr}(E))$ is finite or equivalently, if and only if $C_{\mathrm{GI}(2, \mathbb{Z})} F$ is finite.

Consequently, $\operatorname{Out}(E)$ is infinite if and only if its underlying crystallographic group is isomorphic to $\mathbb{Z}^{2}$ or to $\mathbb{Z}^{2} \rtimes_{A} \mathbb{Z}_{2}$, where $A=\left(\begin{array}{cc}-1 & 0 \\ 0 & -1\end{array}\right)$.

Example 4.7. Consider the following family of 3-dimensional almost-Bieberbach groups $E$ (number 8 in [4]). They all are extensions $1 \rightarrow \Gamma_{k} \rightarrow E \rightarrow F \cong \mathbb{Z}_{2} \times \mathbb{Z}_{2} \rightarrow 1$ and have a presentation of the following type

$$
\begin{array}{rlll}
E:<a, b, c, \alpha, \beta \|[b, a]=c^{k} & {[c, a]=1} & {[c, b]=1} \\
\alpha a=a^{-1} \alpha c^{\frac{h}{2}} & \alpha b=b^{-1} \alpha c^{-\frac{h}{2}} & \alpha c=c \alpha \\
\beta a=a \beta & \beta b=b^{-1} \beta c^{-\frac{h}{2}} & \beta c=c^{-1} \beta \\
\alpha^{2}=c & \beta^{2}=a & \alpha \beta=a^{-1} b^{-1} \beta \alpha c^{-1-\frac{h}{2}}
\end{array}
$$


where $k \in 4 \mathbb{Z}_{0}$. Obviously, the underlying crystallographic group of $E$ is presented by

and

$$
\begin{aligned}
\operatorname{Cr}(E):\langle a, b, \alpha, \beta \|[b, a]=1 & \alpha a=a^{-1} \alpha & \alpha b=b^{-1} \alpha \\
\beta a=a \beta & \beta b=b^{-1} \beta & \\
\alpha^{2}=1 & \beta^{2}=\alpha & \alpha \beta=a^{-1} b^{-1} \beta \alpha
\end{aligned}
$$

$$
C_{\mathrm{Gl}(2, \mathbb{Z})}\left(\mathbb{Z}_{2} \times \mathbb{Z}_{2}\right)=\left\{\left(\begin{array}{ll}
1 & 0 \\
0 & 1
\end{array}\right),\left(\begin{array}{cc}
1 & 0 \\
0 & -1
\end{array}\right),\left(\begin{array}{cc}
-1 & 0 \\
0 & 1
\end{array}\right),\left(\begin{array}{cc}
-1 & 0 \\
0 & -1
\end{array}\right)\right\} .
$$

Hence $\operatorname{Out}(E)$ is finite.

Indeed, if we apply the systematical method to study Out $(E)$ explained in [8], we obtain a short exact sequence

$$
1 \rightarrow \mathbb{Z}_{2} \rightarrow \operatorname{Out}(E) \rightarrow \mathscr{D}_{4} \rightarrow 1
$$

where $\operatorname{Out}(E)$ is generated by the images of the following $E$-automorphisms:

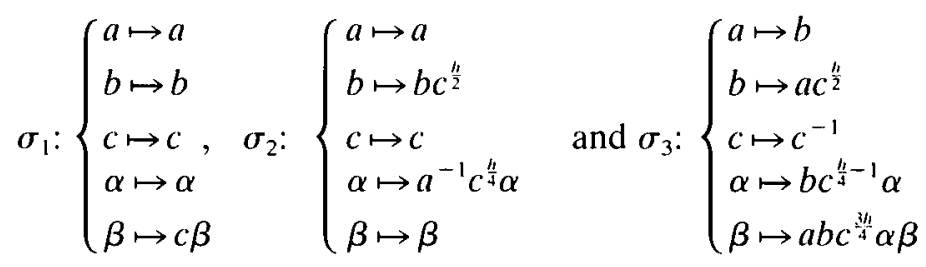

or $\operatorname{Out}(E) \cong \mathbb{Z}_{2} \times \mathscr{D}_{4}$.

4.3.2. Almost-crystallographic groups of dimension $>3$. Unfortunately, in higher dimensions, the situation becomes more difficult as the following example (a 4dimensional almost-Bieberbach group of type 76 in [2]) illustrates.

EXAMPLE 4.8. Consider the group $E$ with presentation

$$
\begin{aligned}
& E:\left\langle a, b, c, d, \alpha \|[b, a]=d^{3} \quad[c, a]=1 \quad[c, b]=1\right\rangle . \\
& {[d, a]=1 \quad[d, b]=1 \quad[d, c]=1} \\
& \alpha a=b \alpha \quad \alpha b=a^{-1} \alpha \quad \alpha c=c \alpha \\
& \alpha d=d \alpha \quad \alpha^{4}=c
\end{aligned}
$$

$E$ fits into an essential extension $1 \rightarrow N \rightarrow E \rightarrow \mathbb{Z}_{4} \rightarrow 1$ where

$$
\begin{aligned}
& N:\left\langle a, b, c, d \|[b, a]=d^{3} \quad[c, a]=1 \quad[c, b]=1\right\rangle . \\
& {[d, a]=1 \quad[d, b]=1 \quad[d, c]=1}
\end{aligned}
$$

The isolator $\sqrt[N]{\gamma_{2}(N)}$ is generated by $d$ and hence, the underlying crystallographic group of $E$ is 3-dimensional. One easily verifies that

$$
C_{\mathrm{Gl}(3, \mathbb{Z})} \mathbb{Z}_{4}=\left\{\left(\begin{array}{ccc}
p & q & 0 \\
-q & p & 0 \\
0 & 0 & r
\end{array}\right) \| p, q, r \in\{-1,1\}\right\} \cong \mathbb{Z}_{2} \times \mathbb{Z}_{2} \times \mathbb{Z}_{2}
$$

or $\operatorname{Out}(\operatorname{Cr}(E))$ is finite. 
Consider the following $N$-automorphisms:

$$
v_{z}:\left\{\begin{array}{l}
a \mapsto a \\
b \mapsto b \\
c \mapsto c d^{z}, \quad z \in \mathbb{Z} . \\
d \mapsto d
\end{array}\right.
$$

Obviously, the unipotent automorphism $v_{z}$ belongs to $T=\operatorname{Ker}(D) \cap \operatorname{Ker}\left({ }^{*}\right)$. On the other hand, for each $z \in \mathbb{Z}, v_{z} \notin \operatorname{Inn}(N)$ or the index $\operatorname{of} \operatorname{Inn}(N)$ in $T$ is not finite.

We can lift these $N$-automorphisms $v_{z}(z \in \mathbb{Z})$ to automorphisms of $E$ :

$$
\sigma_{z}:\left\{\begin{array}{l}
a \mapsto a \\
b \mapsto b \\
c \mapsto c d^{z}, \quad z \in \mathbb{Z} . \\
d \mapsto d \\
\alpha \mapsto d \alpha
\end{array}\right.
$$

We verify that $\sigma_{z} \notin \operatorname{Inn}(E)$ and hence determines a free abelian subgroup $\mathbb{Z}$ of outer automorphisms (or Out $(E)$ is infinite).

5. Infra-nilmanifolds supporting an affine diffeomorphism which is not virtually unipotent. From now on, let $M=E \backslash L$ be an infra-nilmanifold associated with the essential extension $1 \rightarrow N \rightarrow E \rightarrow F \rightarrow 1$. The connected, simply connected nilpotent Lie group $L$ is the Mal'cev completion of $N$. An affine diffeomorphism $f \in \operatorname{Aff}(M)$ is called virtually unipotent if and only if there is a lifting $\tilde{f} \in N_{\mathrm{Afr}(L)} E$ of $f$ such that $\mu(\tilde{f}): L \rightarrow L$ is virtually unipotent. We shall prove that, if there is an affine diffeomorphism on an infra-nilmanifold which is not virtually unipotent, then the outer automorphism of the corresponding almost-Bieberbach group is infinite.

An interesting sufficient condition to conclude that the outer automorphism group of an almost-crystallographic group is infinite is the following:

Proposition 5.1. Assume $E$ is an almost-crystallographic group fitting into an essential extension $1 \rightarrow N \rightarrow E \rightarrow F \rightarrow 1$. Write $\psi: F \rightarrow \operatorname{Out}(N)$ for the induced finite, faithful abstract kernel. If there exists an automorphism of $N$ which is not virtually unipotent and corresponding to an outer automorphism in $C_{\mathrm{Out}(N)} \psi(F)$, then $\operatorname{Out}(E)$ is infinite.

Proof. Let $v$ be an automorphism of $N$ which is not virtually unipotent and $p(v) \in C_{\mathrm{Ou}(N)} \psi(F)$. Since the inner automorphism of $N$ induce unipotent matrices, it follows that $p(v)$ has infinite order and hence $\operatorname{Out}(E)$ is infinite (Proposition 3.1).

In the spirit of the previous section, it is interesting to remark that the following result states that the existence of an infinite order automorphism of an almost-Bieberbach group, arising from an automorphism of its translation subgroup which is not virtually unipotent (as introduced in Proposition 5.1), always implies that the outer automorphism group of the underlying crystallographic group is also infinite.

Proposition 5.2. Let $1 \rightarrow N \rightarrow E \rightarrow F \rightarrow 1$ be an essential extension of a $c$-step nilpotem group $N$ and realising an abstract kernel $\psi: F \rightarrow \operatorname{Out}(N)$. Take $\Gamma_{i}=$ $\sqrt[v]{\gamma_{i+1}(N)}(1 \leq i \leq i)$. Consider $D_{i}: \operatorname{Aut}(N) \rightarrow \operatorname{Aut}\left(N / \Gamma_{i}\right)$ as introduced above. Assume $v$ is an automorphism of $N$ such that its image in $\operatorname{Out}(N)$ belongs to $C_{\mathrm{Out}(N)} \psi(F)$.

If, for some $j(1 \leq j \leq c)$, the $N / \Gamma_{j}$-automorphism $D_{j}(v)$ is not virtually unipotent, then, for all $i, 1 \leq i \leq c, D_{i}(v)$ is not virtually unipotent and hence all the outer 
automorphism groups of the almost-crystallographic groups $E / \Gamma_{i}$ (and in particular of the underlying crystallographic group $\mathrm{Cr}(E)$ ) are infinite.

Proof. If $\Gamma_{i}=\sqrt[N]{\gamma_{i+1}(N)}(1 \leq i \leq c)$, all extensions $1 \rightarrow N / \Gamma_{i} \rightarrow E / \Gamma_{i} \rightarrow F \rightarrow 1$ are essential and hence induce faithful abstract kernels $\psi_{\Gamma_{i}}: F \leftarrow \operatorname{Out}\left(N / \Gamma_{i}\right)$. Let $v \in \operatorname{Aut}(N)$ such that $p(v) \in C_{\operatorname{Out}(N)} \psi(F)$. Then, for all $i, \bar{D}_{i}(v) \in C_{\operatorname{Out}\left(N / \Gamma_{i}\right)} \psi_{\Gamma_{i}}(F)$. If, for some $j$ $(1 \leq j \leq c), D_{j}(v)$ is not virtually unipotent, then $D_{j}(v)$ has an eigenvalue $\lambda$ of absolute value not one and hence, for all $i, j \leq i \leq c, D_{i}(v)$ is also not virtually unipotent (since $D_{i}(v)$ also has $\lambda$ as eigenvalue).

Consequently, $v\left(=D_{c}(v)\right)$ itself is not virtually unipotent and hence, because of Lemma $1.2, D_{1}(v)$ is also not virtually unipotent or we can assume that $j=1$. Hence all outer automorphism groups $\operatorname{Out}\left(E / \Gamma_{i}\right)(1 \leq i \leq c)$ are infinite (Proposition 5.1).

For infra-nilmanifolds (or almost-Bieberbach groups), we now establish the following important equivalence:

Proposition 5.3. Assume $M=E \backslash L$ is an infra-nilmanifold associated with an essential extension $1 \rightarrow N \rightarrow E \rightarrow F \rightarrow 1$. Let $\psi: F \rightarrow \operatorname{Out}(N)$ be the induced abstract kernel. Then the following are equivalent:

1. M supports an affine diffeomorphism which is not virtually unipotent.

2. There is an automorphism of $E$ restricting to an $N$-automorphism which is not virtually unipotent.

3. There is an $N$-automorphism which is not virtually unipotent in $\mathcal{M}_{\psi}$ (the inverse image in $\operatorname{Aut}(N)$ of the normaliser of $\psi(F)$ in $\operatorname{Out}(N))$.

4. There is an automorphism of $N$ which is not virtually unipotent and corresponding to an outer automorphism which commutes with $\psi(F)$.

Proof. Assume $f=q(\tilde{f}) \in \operatorname{Aff}(M)$ such that $\tilde{f} \in N_{\mathrm{Aff}(L)} E$ induces, via conjugation, an automorphism of $L$ which is not virtually unipotent. Then the $E$-automorphism induced by $\tilde{f}$ (again via conjugation) of course restricts to an $N$-automorphism which is not virtually unipotent.

Let $\sigma$ be an automorphism of $E$ such that its restriction $A(\sigma)$ to $N$ is not virtually unipotent. Then $A(\sigma)$ (which belongs to $\mathcal{M}_{\psi, a}(1)$ ) is an element of $\mathcal{M}_{\psi}$ which is not virtually unipotent.

Assume $v \in \mathcal{M}_{\psi}$ is not virtually unipotent. Since $F$ is finite, the $F$-automorphism $v^{*}$ has finite order, say $k \in \mathbb{N}_{0}$. Consequently, $v^{k}$ is an $N$-automorphism in $\mathcal{M}_{\psi} \cap \operatorname{Ker}\left(^{*}\right)$, which is not virtually unipotent, and, because of (5), corresponding to an outer automorphism belonging to $C_{\text {Out }(N)} \psi(F)$.

Conversely, let $v$ be an automorphism of $N$ which is not virtually unipotent and such that $p(v) \in C_{\text {Out }(N)} \psi(F)$. Since $\mathcal{M}_{\psi, a}$ has finite index in $\mathcal{M}_{\psi}\left(F\right.$ is finite), there is a $k \in \mathbb{N}_{0}$ such that $v^{k} \in \mathcal{M}_{\psi, a}$ and $v^{k}$ is of course still not virtually unipotent. Because of (1), there is an $E$-automorphism $\sigma$ restricting to $v^{k}$ on $N$. It is well known that $\sigma$ can be realised as an affine conjugation in $\operatorname{Aff}(L)$, say determined by $\tilde{f} \in N_{\mathrm{Afr}(L)} E$. Then the affine diffeomorphism $f=q(\tilde{f}) \in \operatorname{Aff}(M)$ is, by definition, not virtually unipotent.

Combining this proposition with Proposition 5.1 and 5.2, we obtain

THEOREM 5.4. If there is an automorphism of an almost-Bieberbach group $E$ restricting to an automorphism of its translation subgroup which is not virtually unipotent, 
then $\operatorname{Out}(E)$ (and the outer automorphism group of the underlying crystallographic group $\operatorname{Cr}(E)$ of $E)$ are infinite.

Equivalently, we may write this as follows.

THEOREM 5.5. If an infra-nilmanifold $M$ supports an affine diffeomorphism which is not virtually unipotent, then $\operatorname{Out}\left(\pi_{1}(M)\right)$ is infinite.

Note. Here we should refer to the work of H. L. Porteous ([18]), J. Franks ([7]), A. Manning ([16]),... on Anosov diffeomorphisms of resp. flat Riemannian manifolds and infra-nilmanifolds. These diffeomorphisms are induced by (in fact, topologically conjugated to ([16])) hyperbolic (this means with no eigenvalues of absolute value one) automorphisms of the universal covers, which are called resp. Anosov automorphisms ([18]) and hyperbolic infra-nilmanifold automorphisms ([7], [16]). In our terminology, for an infra-nilmanifold $M=E \backslash L$, a hyperbolic infra-nilmanifold automorphism is an affine diffeomorphism of $M$ inducing a hyperbolic automorphism of $L$. This is of course closely related to our work: indeed, remark that a hyperbolic automorphism of $L$ is clearly not virtually unipotent. In this perspective, with a proof completely similar to the one for Proposition 5.3, one easily shows that

Proposition 5.6. An infra-nilmanifold $M=E \backslash L$ associated with an essential extension $1 \rightarrow N \rightarrow E \rightarrow F \rightarrow 1$ and an abstract kernel $\psi: F \rightarrow \operatorname{Out}(N)$ supports a hyperbolic infranilmanifold automorphism if and only if there exists a hyperbolic $N$-automorphism corresponding to an outer automorphism which commutes with $\psi(F)$.

This generalises [18, Theorem 1.1].

Obviously, if there is a hyperbolic infra-nilmanifold automorphism, then the infra-nilmanifold also admits an affine diffeomorphism which is not virtually unipotent. Hence

COROLLARY 5.7. If an infra-nilmanifold $M$ supports an Anosov diffeomorphism, then $\operatorname{Out}\left(\pi_{1}(M)\right)$ is infinite.

This is a generalisation of the similar result, presented in [20], for flat Riemannian manifolds.

Let us illustrate these results with an example:

EXAMPLE 5.8. Consider the Heisenberg group

$$
H=\left\{\left(\begin{array}{ccc}
1 & y & z \\
0 & 1 & x \\
0 & 0 & 1
\end{array}\right) \mid x, y, z \in \mathbb{R}\right\}
$$

which is connected, simply connected and nilpotent of class 2 . In $H$ consider

$$
A=\left(\begin{array}{lll}
1 & 0 & 0 \\
0 & 1 & 1 \\
0 & 0 & 1
\end{array}\right), \quad B=\left(\begin{array}{lll}
1 & 1 & 0 \\
0 & 1 & 0 \\
0 & 0 & 1
\end{array}\right), \quad C=\left(\begin{array}{lll}
1 & 0 & 1 \\
0 & 1 & 0 \\
0 & 0 & 1
\end{array}\right)
$$

So, $H=\left\{A^{*} B^{y} C^{z} \| x, y, z \in \mathbb{R}\right\}$ and observe that, in $H,[B, A]=C,[C, A]=1$ and $[C, B]=1$. Let $\mathfrak{h}$ indicate the corresponding Lie algebra. Then $\log A, \log B$ and $\log C$ form a suitable basis of $\mathfrak{h}$ (as vectorspace). 
Take the uniform lattice $N$ in $H$ generated by $a=A, b=B$ and $c=\sqrt{C}$. As a presentation for $N$, we have

$$
N:\left\langle a, b, c \|[b, a]=c^{2},[c, a]=[c, b]=1\right\rangle .
$$

Take $F \cong \mathbb{Z}_{2}$, given as $\{1, \alpha\}$, and let $F$ act on $H$ via $\varphi: F \rightarrow \operatorname{Aut}(H)$ such that $\alpha$ sends $A \mapsto A^{-1}, B \mapsto B^{-1}$ and $C \mapsto C$. Let $E$ be presented by

$$
\left.\begin{array}{rlll}
E:\left\langle a, b, c, \alpha \|[b, a]=c^{2}\right. & {[c, a]=1} & {[c, b]=1}
\end{array}\right) .
$$

It is not hard to see that $E$ is a 3-dimensional almost-Bieberbach group fitting into an essential extension $1 \rightarrow N \rightarrow E \rightarrow \mathbb{Z}_{2} \rightarrow 1$ realising a faithful abstract kernel $\psi: \mathbb{Z}_{2} \stackrel{\varphi}{\rightarrow}$

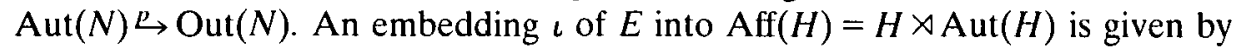

$$
\iota: E \hookrightarrow \operatorname{Aff}(H): a \mapsto(A, 1), b \mapsto(B, 1), c \mapsto(\sqrt{C}, 1), \alpha \mapsto(\sqrt[4]{C}, \varphi(\alpha)) .
$$

Let $M=E \backslash H$ be the corresponding 3-dimensional infra-nilmanifold.

Take $(\sqrt{B}, \beta)$ in Aff $(H)$, where $\beta \in \operatorname{Aut}(H)$ sends $A \mapsto A B \sqrt{C}, B \mapsto A$ and $C \mapsto C^{-1}$. One easily verifies that the conjugation determined by $(\sqrt{B}, \beta)$ sends

$$
\begin{array}{ll}
\iota(a)=(A, 1) & \mapsto(A B C, 1)=\iota\left(a b c^{2}\right) \\
\iota(b)=(B, 1) & \mapsto(A \sqrt{C}, 1)=\iota(a c) \\
\iota(c)=(\sqrt{C}, 1) & \mapsto\left(\sqrt{C^{-1}}, 1\right)=\iota\left(c^{-1}\right) \\
\iota(\alpha)=(\sqrt[4]{C}, \varphi(\alpha)) & \mapsto\left(B \sqrt{C^{-1}} \sqrt[4]{C}, \varphi(\alpha)\right)=\iota\left(b c^{-1} \alpha\right)
\end{array}
$$

or $(\sqrt{B}, \beta) \in N_{\mathrm{Aft}(H)} E$. Write $f$ for the corresponding affine diffeomorphism of $M .(\sqrt{B}, \beta)$ induces an automorphism $\sigma$ of $E$ given by

$$
\begin{aligned}
\sigma: E & \rightarrow E \\
a & \mapsto a b c^{2} \\
b & \mapsto a c \\
c & \mapsto c^{-1} \\
\alpha & \mapsto b c^{-1} \alpha
\end{aligned}
$$

Write $v$ for the restriction of $\sigma$ to $N$ and $\tilde{v}$ for the unique lift to $\operatorname{Aut}(H)$ sending $A \mapsto A B C, B \mapsto A \sqrt{C}$ and $C \mapsto C^{-1}$. The reader can verify that $\mu(b) \circ \varphi(a) \circ v=v \circ \varphi(a)$ on $N$ or $p(v) \in C_{\mathrm{Out}(N)} \psi\left(\mathbb{Z}_{2}\right)$.

Via the Campbell-Baker-Hausdorff formula, we compute that $d \tilde{v} \in \operatorname{Aut}(\mathfrak{h})$ maps

$$
\left\{\begin{array}{l}
\log A \mapsto \log (A B C)=\log A+\log B+\frac{1}{2} \log C \\
\log B \mapsto \log (A \sqrt{C})=\log A+\frac{1}{2} \log C \\
\log C \mapsto \log \left(C^{-1}\right)=-\log C
\end{array}\right.
$$

and hence corresponds in $\operatorname{Gl}(3, \mathbb{R})$ to

$$
A_{v}=\left(\begin{array}{ccc}
1 & 1 & 0 \\
1 & 0 & 0 \\
\frac{1}{2} & \frac{1}{2} & -1
\end{array}\right)
$$


Compute that the characteristic polynomial of $A_{v}$ equals $-x^{3}+2 x+1$ (note that all coefficients are indeed integers) and the eigenvalues of $A_{v}$ are $-1, \frac{1-\sqrt{5}}{2}$ and $\frac{1+\sqrt{5}}{2}$. Hence, the affine diffeomorphism $f$ of $M$ is not virtually unipotent (but not hyperbolic or $f$ is not an Anosov diffeomorphism) and consequently, Out $(E)$ is infinite. Indeed, the reader can verify (use the theoretic set-up developed in [8]) that

$$
1 \rightarrow \mathbb{Z}_{2} \times \mathbb{Z}_{2} \rightarrow \operatorname{Out}(E) \rightarrow \mathrm{Gl}\left(2, \mathbb{Z}_{)}\right) \mathbb{Z}_{2} \rightarrow 1
$$

is exact.

We also could have used Proposition 5.2 to conclude that $\operatorname{Out}(E)$ is infinite since, if we factor out $\Gamma=\sqrt[N]{\gamma_{2}(N)}$ (the subgroup generated by $c$ ), $v$ induces a hyperbolic automorphism $D(v) \in \mathrm{Gl}(2, \mathbb{Z})$ on the abelian level sending $a \mapsto a b$ and $b \mapsto a$. Even more straightforward, $\operatorname{Out}(E)$ is infinite since $\operatorname{Cr}(E) \cong \mathbb{Z}^{2} \rtimes_{A} \mathbb{Z}_{2}$, where $A=\left(\begin{array}{cc}-1 & 0 \\ 0 & -1\end{array}\right)$ (Corollary 4.6).

Acknowledgement. This manuscript was written while the first author was visiting the Institute of Mathematics at the University of Gdańsk. He wishes to thank the director and the staff for their hospitality. The authors are also grateful to Dr. K. Dekimpe for the helpful conversations.

\section{REFERENCES}

1. M. Bridson and S. Gersten, Optimal isoperimeric inequality for torus bundles over the circle. Quart. J. Math. Oxford (2) 47 (1996) 1-23.

2. K. Dekimpe, Almost Bieberbach Groups: cohomology, construction and classification (Doctoral Thesis, K. U. Leuven, 1993).

3. K. Dekimpe, Determining the translation part of the fundamental group of an infrasolvmanifold of type $(R)$, to appear in Math. Proc. Camb. Phil. Soc.

4. K. Dekimpe, P. Igodt, S. Kim and K. B. Lee, Affine structures for closed 3-dimensional manifolds with NIL-geometry, Quart. J. Math. Oxford (2) 46 (1995) 141-167.

5. K. Dekimpe, P. Igodt and W. Malfait, On the Fitting subgroup of almost crystallographic groups, Tijdschrift van het Belgisch Wiskundig Genootschap, B1 (1993) 35-47.

6. K. Dekimpe and W. Malfait, Almost-crystallographic groups with many outer automorphisms, Comm. Algebra 23(8) (1995), 3073-3083.

7. J. Franks, Anosov diffeomorphisms, In: Global Analysis: Proceedings of the Symposia in Pure Mathematics 14 (1970) 61-93.

8. P. Igodt and W. Malfait, Extensions realising a faithful abstract kernel and their automorphisms, Manuscripta Math. 84 (1994) 135-161.

9. P. Igodt and W. Malfait, Representing the automorphism group of an almost crystallographic group, Proc. Amer. Math. Soc. 124(2) (1996) 331-340.

10. Y. Kamishima, K. B. Lee and F. Raymond, The Seifert construction and its applications to infra-nilmanifolds, Quart. J. Math. Oxford (2) 34 (1983) 433-452.

11. K. B. Lee and F. Raymond, Geometric realization of group extensions by the Seifert construction, Contemporary Math. A. M. S., 33 (1984) 353-411.

12. K. B. Lee and F. Raymond, Rigidity of almost crystallographic groups, Contemporary Math. A. M. S. 44 (1985) 73-78.

13. S. Mac Lane, Homology, volume 114 of Die Grundlehren der Math. Wissenschaften (Springer-Verlag: Berlin, Heidelberg, New York, 1975).

14. A. I. Mal'cev, On a class of homogeneous spaces, Translations A. M. S. 39 (1951) 1-33. 
15. W. Malfait, Symmetry of Infra-Nilmanifolds: An Algebraic Approach (Doctoral Thesis, K. U. Leuven, 1994).

16. A. Manning, There are no new Anosov diffeomorphisms on tori, Amer. J. Math. 96(3) (1974) 422-429.

17. W. Parry, ergodic peoperties of affine transformations and flows on nilmanifolds, Amer. J. Math. 91 (1969) 757-771.

18. H. L. Porteous, Anosov dffeomorphisms of flat manifolds, Topology, 11 (1972) 307-315.

19. I. Stewart and D. Tall, Algebraic Number Theory, second edition (Chapman and Hall Mathematics Series, 1987).

20. A. Szczepański, Outer automorphism groups of Bieberbach groups, to appear in Bull. of Belg. Math. Soc. (Simon Stevin), 1996.

Wim MaLfaIT

Katholieke Universiteit Leuven Campus Kortrijk

Universitaire Campus

B-8500 KORTRIJK (Belgium)

E-mail: Wim.Malfait@kulak.ac.be

ANDRZEJ SZCZEPAŃSKI

INSTITUTE OF MATHEMATICS

UNIVERSITY OF GDAŃSK

UL. WITA Stwosza 57

80-952 GDANSK (Poland)

E-mail: matas@paula.univ.gda.pl 